\title{
The Effect of Macroscopic and Microscopic Patterns of Stainless Steel Surface on the Efficiency of Dropwise Condensation and Precipitation
}

\author{
Fatema Tuz Zohra, Md Muzaffer Hosen Akanda and Bahram Asiabanpour
}

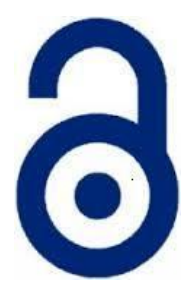

\author{
Received: 28 February 2021 \\ Accepted: 29 April 2021 \\ Published: 15 July 2021 \\ Publisher: Deer Hill Publications \\ (C) 2021 The Author(s) \\ Creative Commons: CC BY 4.0
}

\begin{abstract}
Atmospheric water generator (AWG) technologies are considered a potential new source of freshwater. Thermoelectric (TEC) dehumidifiers are one of this technology where it uses the Peltier effect to create a heat flux at the junction of two different materials. This device is well known for its thermoelectric cooling application, from as small as portable beverage coolers to as big as submarines. The problems of using this device are ice buildup and relatively low efficiency. If the surface of the material can be engineered in a way that the dropwise condensation, as well as precipitation from the cooling plate, is enhanced, it is possible to maximize the efficiency of TEC-driven AWG with minimum energy consumption. This study investigates the effects of macroscopic (bump/dent, patterns that are visible to the human eye) and microscopic patterns (surface finish, quality, grain distribution, etc. visible under a microscope) on industry-grade stainless (SS) surfaces on dropwise condensation and precipitation. The surface contact angle is considered here to understand the hydrophobic/hydrophilic properties of the surfaces before and after applying hydrophobic coating under constant ambient conditions. Better droplet nucleation and quicker precipitation have been observed on the surfaces after treating a hydrophilic and hydrophobic surface with hydrophobic spray. It can be inferred that even though the initial surface property of the two samples were different, it was possible to achieve similar level of super hydrophobicity after applying the spray. This proves that surfaces can be modified with appropriate treatment to achieve desired properties.
\end{abstract}

Keywords: Dropwise condensation, hydrophobic and hydrophilic surface, macroscopic pattern, microscopic pattern.

\section{INTRODUCTION}

For dropwise condensation to occur, a droplet has to nucleate, grow, merge with surrounding droplets, and finally detach from the surface leaving space for more droplet accumulation (1)(2). The factors that affect this condensation process are many, such as surrounding temperature and relative humidity, surface property (surface energy, contact angles), angle of tilt of the surface relative to gravity, surface chemistry, groove geometry to aid the droplet motion (3), etc. Hydrophilic surfaces are the ones that attract water and helps wetting surfaces and the hydrophobic ones repel water so the surface becomes self-cleaning and non-adhesive to water. The contact angle is a useful measure to identify hydrophilic or hydrophobic surfaces by an instrument called a goniometer. The angle of contact between the dispersed liquid drops and the surface indicates this particular surface property as shown in Fig. 1 (a) (4).

Hence, a surface needs to possess the perfect property based on the application. For example, during dropwise condensation, a surface must be hydrophilic enough to be able to attract moisture to initiate droplet formation but hydrophobic enough so that it leaves the surface quickly to leave space for new droplet formation. While the microscopic structure is responsible for a surface to be either hydrophilic or hydrophobic, both the micro and the macroscopic texture (bump, dent, groove, etc. that are visible to the human eye without any microscope) on a surface can be helpful to guide the coalescence and release of the droplets with the aid of gravity (5). The presence of the ripples (Fig. 1 (c), (d)) creates geometric confinement, and thus condensed droplets follow the orientation of textured features as shown in Fig. 1 (b).

Numerous studies have been conducted to determine the effect of both chemical and geometric patterns on wetting characteristics and the motion of condensed droplets positioned horizontally. Stainless steel (SS), an ironbased alloy is one of the most commonly used materials in a diverse industry because of its superior physical and

F. T. Zohra, M. H. Akanda and B. Asiabanpour $区$

Ingram School of Engineering

Texas State University

San Marcos, TX 78666 USA

E-mail: ba13@txtstate.edu

Reference: Zohra et al. (2021). The Effect of Macroscopic and Microscopic Patterns of Stainless Surface on the Efficiency of Dropwise Condensation and Precipitation. International Journal of Engineering Materials and Manufacture, 6(3), 163-169. 
mechanical properties as well as excellent anti-bacterial and corrosion resisting qualities (6)(7)(8). This metal is often used as a substrate to create either super hydrophilic or superhydrophobic surfaces based on the application requirements (8). This surface property depends somewhat on the surface finish by manufacturers and is usually found hydrophilic. Different procedures, such as plasma, laser, physical \& chemical etching, machining, and coatings, etc. are used to alter the wettability of SS to obtain desired properties. AISI 304 (also known as SAE 304) SS has the composition of different elements (9) as shown in Fig. 2. Contact angle and wettability are proven to be impacted greatly by a wide range of roughness introduced to the substrate, such as rain resistance porous clothing (10) (11) (12)(13).

Other studies show that the wetting properties of stainless steel (SS) plates treated with atmospheric pressure plasma (APP) using three different parameters (gap distance, nozzle scan velocity, pass number) can contribute to an improvement of wettability and/or adhesion properties of the surface (14).

Biphilic surface- a surface topography of a combination of hydrophobic patterns on hydrophilic structures has been developed and proved to be useful for enhancing dropwise condensation (Fig. 3 (a-d)) with long-term functionality and possible for large scale production (15). A combination of the hydrophobic surface with super hydrophilic grooves on stainless steel showed better condensation heat transfer than each of them separately (Fig. $3(e)$ ) (16). This hybrid surface showed effective control of droplet sizes with an optimum grid spacing of a super hydrophilic network.

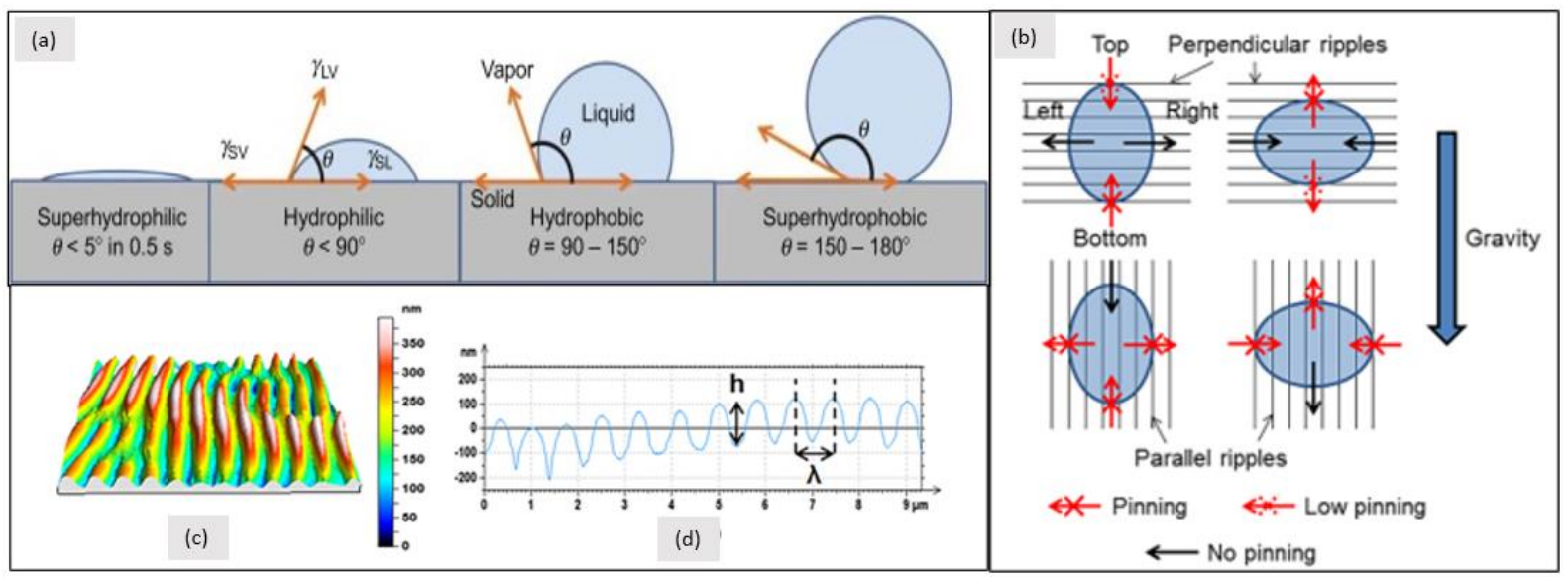

Figure 1: Schematic diagram representing (a) water contact angle on hydrophilic, hydrophobic, and superhydrophobic surfaces (4). (b) a proposed mechanism that could explain the droplet relaxation on samples textured with ripples and fixed on vertical support. (c) AFM scan (size: $10 \times 10 \mu \mathrm{m}^{2}$ ) of ripples replicated at $75 \%$ on polycarbonate. (d) A corresponding height profile of the AFM scan is presented in (c). Source for (b), (c), (d) (5).

\begin{tabular}{|l|l|l|l|l|l|l|l|l|}
\hline Standard & AISI (UNS) & $\mathrm{C}, \leq$ & $\mathrm{Si}, \leq$ & $\mathrm{Mn}, \leq$ & $\mathrm{P}, \leq$ & $\mathrm{S}, \leq$ & $\mathrm{Cr}$ & $\mathrm{Ni}$ \\
\hline ASTM A276/A276M & $304(\mathrm{~S} 30400)$ & 0.08 & 1.00 & 2.00 & 0.045 & 0.030 & $18.0-20.0$ & $8.0-11.0$ \\
\hline
\end{tabular}

Figure 2: Composition of Stainless Steel (9).
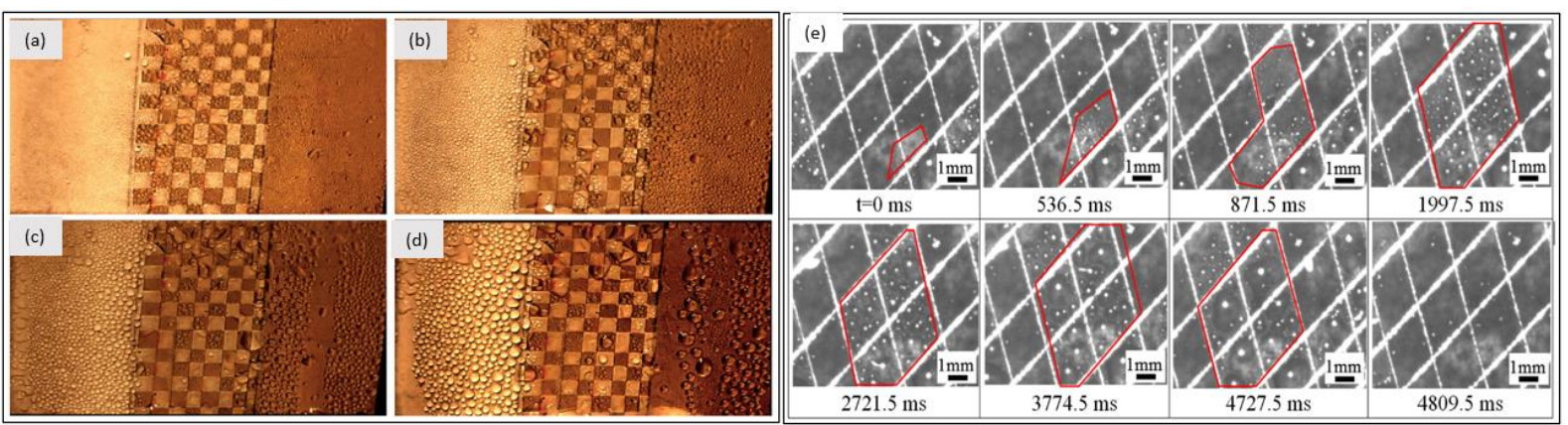

Figure 3: The comparison of the time evolution of condensation process (a-d) on hydrophobic (left), $2 \mathrm{~mm}$ biphilic (center), and hydrophilic (right) regions. The images are taken every $10 \mathrm{~min}$. The biphilic substrate shows better droplet nucleation and removal throughout the experiment (15). (e) growth, movement, and sweeping of droplets on Hydrophobic SS surface with super hydrophilic grooves. 


\section{EXPERIMENTAL PROCEDURES}

In this work, we collected industry-grade SS samples that are already being mass-manufactured with diverse patterns (with biggest bumps to smallest) and surface finish and investigated the surface wettability with precipitation experiment. The as-received samples ( $\left.3^{\prime \prime} \times 3^{\prime \prime}\right)$ were at first cleaned with isopropanol to remove any contamination and visually observed with the help of the naked eye and optical microscope. Contact angles were measured using a goniometer and dropwise condensation and precipitation experiments were conducted using a humidifier. The stainless-steel plates were held at a $30^{\circ}$ angle to the gravity and $90^{\circ}$ to the incoming mist to ensure the maximum amount of mist capture (Fig. 4). Photographs and videos were taken for future reference during analysis. After this first step, the most hydrophilic and the most hydrophobic samples were identified. In the second stage, these two samples were used to carry out the further experiment. A commercial-grade hydrophobic spray is used to coat these samples instead of any expensive machining or chemical etching and similar procedures were carried out as in the first step.

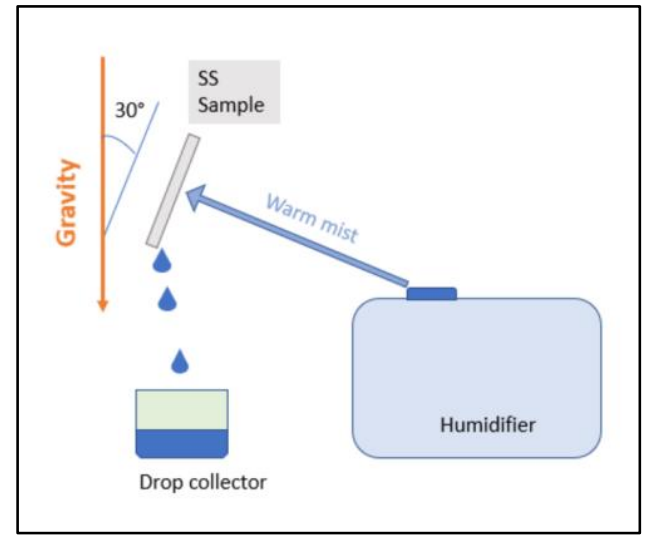

Figure 4: Experimental setup for condensation, droplet growth, and precipitation.

\section{RESULTS}

\subsection{Step - 1:}

In this step, the samples are cleaned and observed under an optical microscope, contact angles were measured, and finally, a precipitation experiment was done using the warm mist from a commercial humidifier.

\subsubsection{Visual Examination}

The photographs of SS samples in as-received condition, enlarged view of a specific section of the samples, photographed under the optical microscope on the scale of $10 \mu \mathrm{m}$, and photographs of measured contact angle of each sample by goniometer with $5 \mu \mathrm{L}$ droplet are shown in Fig. 5 . These samples were randomly chosen and organized from the biggest bump two no bump. Sample 1 having the biggest and highest diamond-shaped bump to sample 8 that has no macroscopic bump at all. Each sample was cleaned with Isopropanol and let dry for 30 minutes before use. According to the manufacturer, all the samples had a 'Satin' surface finish which is a general-purpose bright polish commonly used for architectural applications. For samples 1 to 5 , macroscopic patterns are not visible under microscope view Since the scale is smaller than each bump. From samples 6 to 8, the microscopic view shows the surface structures and finishes more clearly and distinctively. As shown in this figure, sample- 4 having a squareshaped microscopic pattern shows the highest contact angle of $102^{\circ}$, and sample-7 having a tiny Oval shaped microscopic pattern shows the lowest contact angle of approximately $38^{\circ}$. For sample-1, the macroscopic bump was visible even under the goniometer, so the surface on which the droplet is sitting doesn't look fairly flat. For sample3 , having a circular pyramid-shaped bond on the surface, it was impossible for putting a droplet steady on the surface to be able to measure its exact contact angle. But visual inspection of the droplet shape and non-adhesive nature of the droplet to the surface proves that it is hydrophobic. 


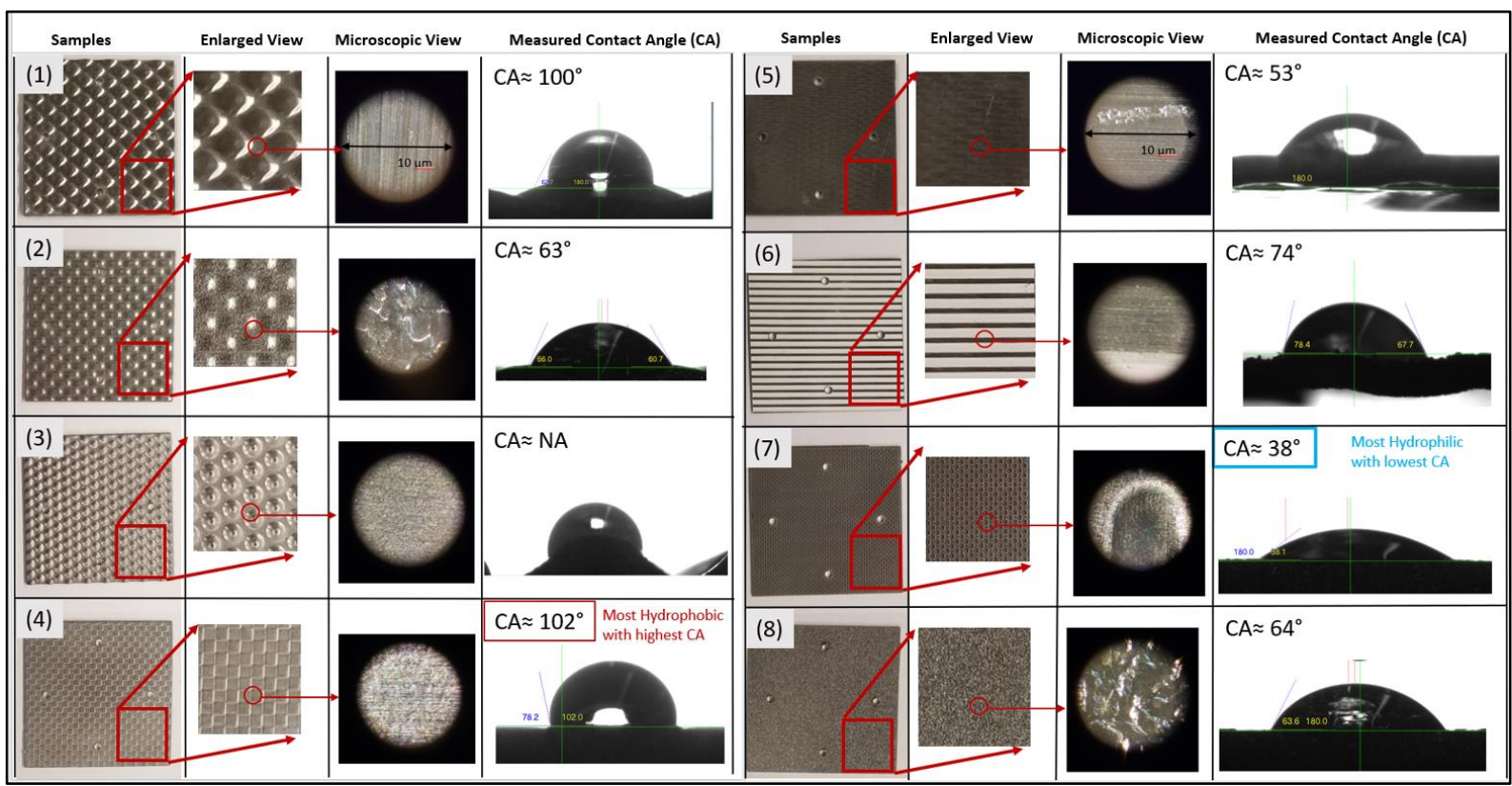

Figure 5: Photograph and enlarged view of the samples, microscopic view, measured contact angle. All the microscopic view has same scale of $10 \mu \mathrm{m}$. Except for the contact angles that were screen shot directly in computer, all the photographs were taken with the camera of Samsung Note 2.

\subsubsection{Precipitation}

Each sample was exposed to warm humidifying mist to acquire condensation, droplet formation, and precipitation under a constant ambient condition (Temperature $22^{\circ} \mathrm{C}$, Relative Humidity $=30 \%$ ). Samples that are categorized as hydrophobic (samples- 1, 3, 4), tends to form droplets and dissipated more often than the hydrophilic ones (samples 2, 5-8). Photographs of time-lapse during this experiment for samples 4 (hydrophobic) \& 7 (hydrophilic) are presented in Fig. 6 for comparison. Hydrophilic surfaces tend to form a thin water film on the surface, thus preventing dry space for new droplet nucleation. Upon close observation, droplets are seen to be formed along with the macroscopic pattern (red diamond in Fig. 6) of the hydrophobic surface (Sample-4) and released at once when the surface energy becomes less than the gravitational pull. Droplets formed on the hydrophilic surface create a thin film together wetting the surface in a circular pattern (blue circle in Fig. 6).

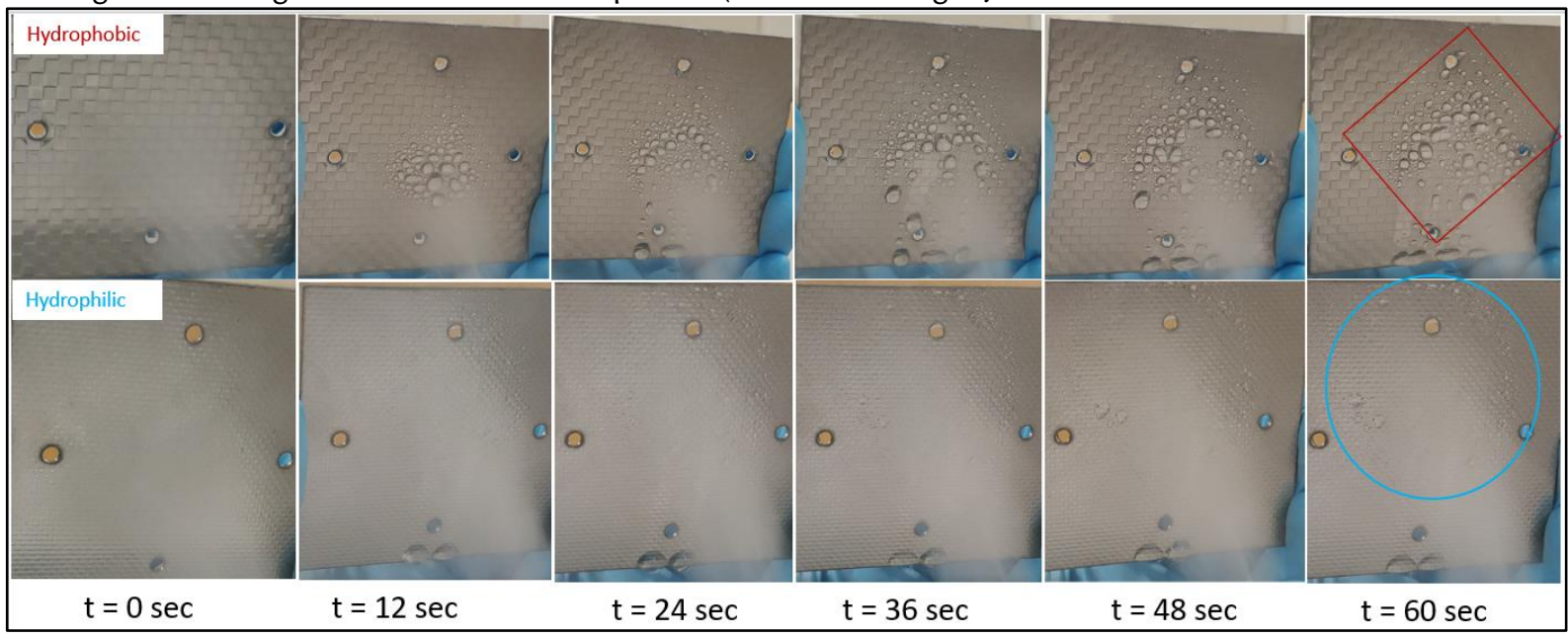

Figure 6: The comparison of time evolution on the droplet growth, movement, and sweeping process on Hydrophobic and hydrophilic SS surface with different macro patterns. All the photographs were taken with the camera of Samsung Note 2. 


\subsection{Step - 2:}

In this step, samples with two extreme contact angles representing hydrophobic (sample-4) and hydrophilic (sample7) are coated with a commercially available superhydrophobic spray (Rust-Oleum Never Wet Step 2 Coat) for further experiments. This coating system creates a moisture repelling barrier on a variety of substrates such as metal, wood, aluminum, galvanized metal, PVC, concrete, masonry, asphalt, vinyl siding, fiberglass, canvas, most plastics and more (17). It also gives the surface a haze finish that slightly alters the color. The sample surfaces are washed with isopropanol to clean any contamination and thoroughly dried. Three coats are applied and let dry for 30 minutes according to the instruction before the contact angle measurement and precipitation experiment is performed. After these experiments, observations under the optical microscope were done for a better understanding of the droplet formation.

\subsection{Results After Hydrophobic Coating}

After the samples were treated with a hydrophobic coating, a slight change of colour on the SS surface was visible because of the dried white coloured spray (Fig. 7).

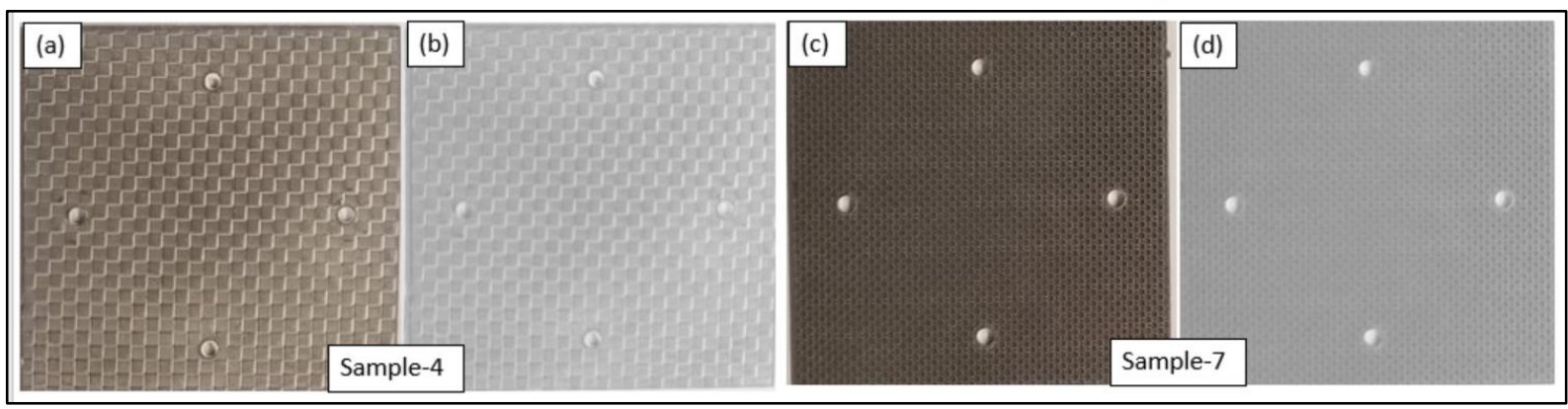

Figure 7: Change of color of the surfaces after applying the hydrophobic spray. Sample-4 (a) before, (b) after, and Sample-7 (c) before, (d) after.

The surfaces showed similar droplet growth, coalescence, and release from the surface which is different than before the coat. Fig. 8 shows these contrasts, the microscopic view of the sample, and their contact angles. Both the surfaces look the same and similar shapes of droplets are found when inspected under a microscope. The contact angle measured for both surfaces is very close and represents a superhydrophobic surface. The adhesion to the surface was so difficult that the droplet size had to be increased from 5 to $15 \mu \mathrm{L}$ to be able to release it on the surface. Because of this superhydrophobic nature, when subjected to the warm mist in the precipitation experiment (at $30^{\circ}$ angle to the gravity and perpendicular to the mist incoming direction), droplets started to fall once they were almost the size of $5 \mu \mathrm{L}$. A much smaller droplet precipitation occurred than that in step-1. The self-cleaning property of the surface improved greatly in the sense that the droplets did not adhere to the surface to form a thin film of water and also macroscopic pattern did not have any effect at this point for guiding the droplets to the bottom of the plate as it did in step-1 for sample-4. 


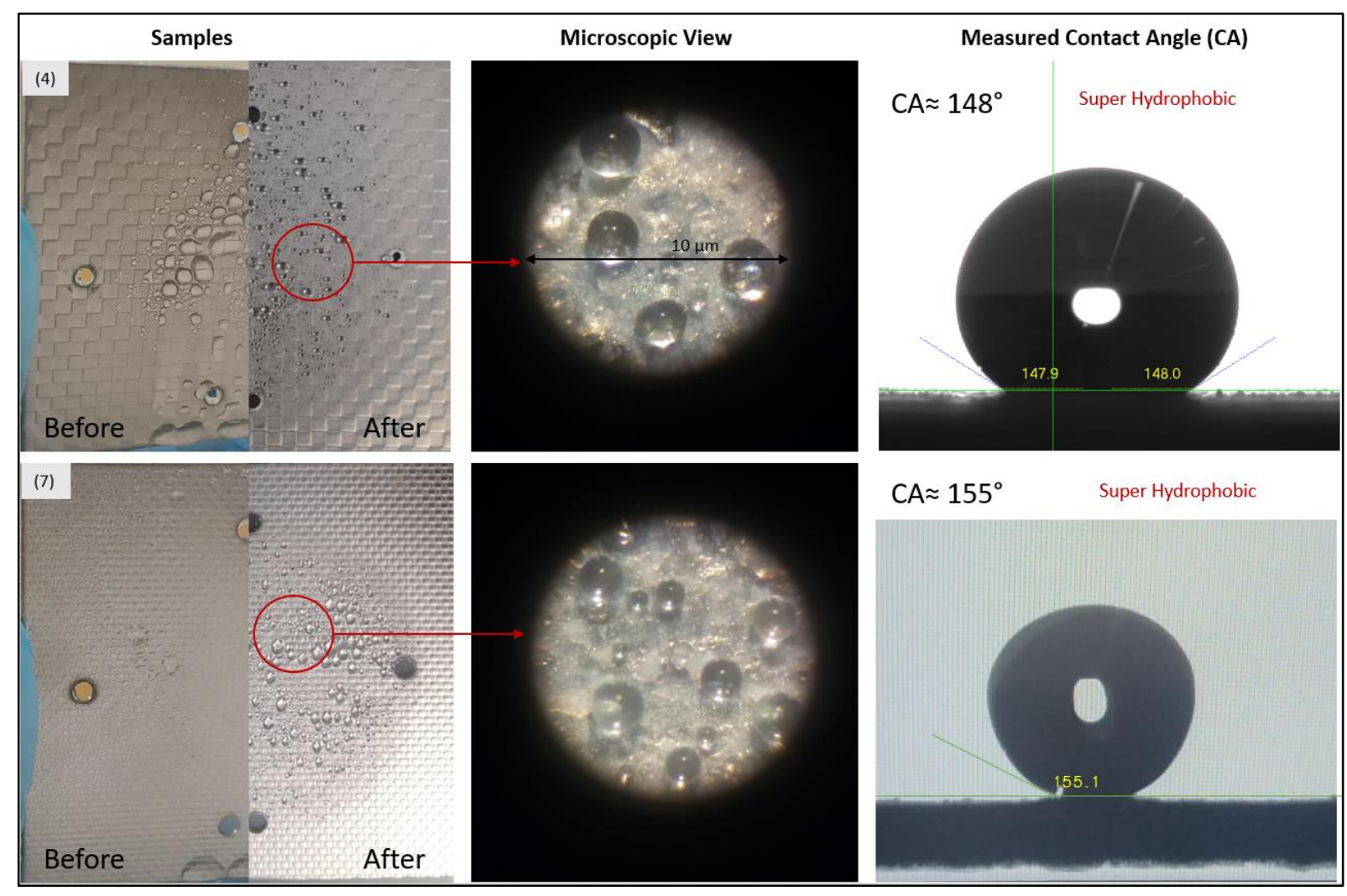

Figure 8: Samples showing photographs of the difference in the wetting of surface before/after applying the hydrophobic spray, droplets under microscope, and contact angle with $15 \mu \mathrm{L}$.

\section{DISCUSSION AND CONCLUSIONS}

Many researchers have studied microscopic patterns and their effects on the hydrophobicity and hydrophilicity of the surfaces. However, not much has been done on the macro geometries and patterns and their integration with micro or nano roughness and material properties. Additionally, the dynamic process (time-based) of AWG may show different results when the system runs long-term.

Our result of observing super hydrophobic property on the samples after treating with hydrophobic spray are consistent with the findings of many previous literatures such as (1)(8)(18) that a superhydrophobic surface enhances both dropwise condensation and condensate droplet departure from that surface. Since the drops in this condensation process are discreet and continually formed and released from the surface, the surface itself gets exposure to facilitate new droplet formation. Whereas, in the filmwise condensation process, the water film always covering the surface acts as a thermal resistance to facilitate the condensation process. Surfaces with this super hydrophobic property also possess some other useful properties such as self-cleaning, anti-icing, wear resistance of the material, etc. Although the coating itself can be easily removed or wiped off clean if needed.

Future research in this area includes systematic categorization of patterns and geometries for the macro features, variation of materials, dynamic droplet size, and computational fluid dynamics to study the flow and heat distribution of the droplet formation over time.

\section{ACKNOWLEDGEMENT}

This work was completed with funding from the US Department of Agriculture (Grant \# 2016-38422-25540). The authors would like to thank the USDA and Texas State University for providing funding and access to both infrastructure and laboratories. The sponsors are not responsible for the content and accuracy of this article. The authors declare that there is no conflict of interest regarding the publication of this paper.

\section{REFERENCE}

1. Dong J, Dong H, Jin Y, Sun L, Ye S, Dong J, et al. Nanograssed Micro-V-Groove Architectures for Continuous Dropwise Condensation and Droplet Directional Movement Exploiting such multiscale coupling effects can help us design optimal con-densation surfaces for high performances of phase-change cooling and. Adv Mater Interfaces [Internet]. 2018 [cited 2021 Apr 10]; Available from: https://doi.org/10.1002/admi.201800202. 
2. Chen X, Wu J, Ma R, Hua M, Koratkar N, Yao S, et al. Nanograssed Micropyramidal Architectures for Continuous Dropwise Condensation. Adv Funct Mater [Internet]. 2011 [cited 2021 Apr 10];21:4617-23. Available from: www.MaterialsViews.com

3. Zhong Y, Jacobi AM, Georgiadis JG. Effects of surface chemistry and groove geometry on wetting characteristics and droplet motion of water condensate on surfaces with rectangular microgrooves. 2012 [cited 2021 Apr 1]; Available from: http://dx.doi.org/10.1016/j.ijheatmasstransfer.2012.10.056

4. Chieng BW, Ibrahim NA, Daud NA, Talib ZA. Functionalization of graphene oxide via gamma-ray irradiation for hydrophobic materials. In: Synthesis, Technology and Applications of Carbon Nanomaterials. Elsevier; 2018. p. 177-203.

5. Pionnier N, Vera J, Contraires E, Benayoun S, Berger R, Valette S. The effect of the orientation and the height of periodic sub-micrometric texturing on dropwise condensation. 2018 [cited 2021 Mar 23]; Available from: https://doi.org/10.1016/j.jcis.2018.04.043

6. Gupta RK, Anandkumar \& B, Choubey A, George \& RP, Ganesh \& P, Upadhyaya \& BN, et al. Antibacterial and Corrosion Studies on Nanosecond Pulse Laser Textured 304 L Stainless Steel Surfaces. [cited 2021 Apr 1]; Available from: https://doi.org/10.1007/s40516-019-00097-9

7. Pramanik A, Basak AK. Stainless steel: Microstructure, mechanical properties and methods of application. Stainless Steel: Microstructure, Mechanical Properties and Methods of Application. Nova Science Publishers, Inc.; 2015. 1-281 p.

8. Cremaldi J, Bhushan B. Fabrication of bioinspired, self-cleaning superliquiphilic/phobic stainless steel using different pathways. J Colloid Interface Sci [Internet]. 2018 [cited 2021 Mar 21]; Available from: https://doi.org/10.1016/j.jcis.2018.02.034

9. SAE 304 stainless steel - Wikipedia [Internet]. [cited 2021 Apr 1]. Available from: https://en.wikipedia.org/wiki/SAE_304_stainless_steel

10. Marmur A. Wetting on hydrophobic rough surfaces: To be heterogeneous or not to be? Langmuir. 2003 Sep 30;19(20):8343-8.

11. Cassie ABD, Baxter S. Wettability of porous surfaces. Trans Faraday Soc. 1944;40:546-51.

12. Dettre RH, Johnson RE. Contact Angle Hysteresis. In: Advances in Chemistry. 1964. p. 136-44.

13. Huerta-Murillo D, García-Girón A, Romano JM, Cardoso JT, Cordovilla F, Walker M, et al. Wettability modification of laser-fabricated hierarchical surface structures in Ti-6Al-4V titanium alloy. Appl Surf Sci. 2019 Jan 1;463:838-46.

14. Kurtuldu F, Altuncu E. Surface Wettability Properties of 304 Stainless Steel Treated by Atmospheric-Pressure Plasma System. In: 4th International Symposium on Innovative Technologies in Engineering and Science. 2016. p. 1350-9.

15. Alizadeh-Birjandi E, Alshehri A, Kavehpour HP. Condensation on Surfaces With Biphilic Topography: Experiment and Modeling. Front Mech Eng. 2019 Jun 25;5.

16. Ji X, Zhou D, Dai C, Xu J. Dropwise condensation heat transfer on superhydrophilic-hydrophobic network hybrid surface. Int J Heat Mass Transf. 2019 Apr 1;132:52-67.

17. Data T. Rust-Oleum Industrial Brands Neverwet ${ }^{\circledR}$ Industrial Gallons.

18. Pandey S. Dropwise and filmwise condensation. Int J Sci Eng Res [Internet]. 2012 [cited 2021 Apr 19];3(4). Available from: http://www.ijser.org. 